\title{
Propagation of waves in a gravitating and rotating anisotropic heat conducting plasma
}

Gebretsadkan W. B.

Department of Physics, College of Natural and Computational Sciences, Mekelle University, P.O. Box 231, Mekelle, Tigrai, Ethiopia (gwbahtas88@yahoo.com).

\begin{abstract}
An inviscid, unbounded, collisionless, gravitating, rotating and heat conducting anisotropic plasma medium which is drifting is considered. The medium is assumed to be embedded in a strong magnetic field. A general dispersion relation is derived using normal mode analysis and its various limiting cases are discussed, compared with similar earlier results for a non-drifting model, and some disagreements are indicated. The dispersion relation reveals the existence of five waves. These different wave modes are discussed in some particular cases analytically. It is found that in the case of parallel propagation all the five waves propagate. When the axis of rotation is across the magnetic field, the modified entropy wave and the modified anisotropic Alfven wave are independent of rotation, gravitation and heat flux. It is shown that the drift velocity has no effect on the stability of these waves but their phase velocities are found to be altered by the drift velocity; the forward propagating modes being increased and the backward modes decreased. The other three waves are affected by gravitation, rotation, drift and parallel component of the heat flux. It is further shown that only two waves propagate in the perpendicular direction. The propagating wave modes in this particular direction are not affected by the drift velocity since wave normal is transverse to the direction of flow.
\end{abstract}

Keywords: Waves, Instabilities, Rotating, Drift, Gravitating, Anisotropy.

\section{INTRODUCTION}

Propagation of waves and instabilities are studied in various collective processes in gaseous plasma because of their possible importance for a number of applications in space and astrophysical plasmas. These plasmas are usually self-gravitating, rotating and embedded in a magnetic field. The gravitational instability is a fundamental concept of modern astrophysical plasma and is connected with the fragmentation of interstellar matter in star formation. It appears to be necessary to investigate the stability of gravitating gas cloud to understand the origin of star formation. Jeans (1902) first studied this instability problem and showed that an infinite, homogeneous, self-gravitating fluid to be unstable for all wave numbers less than the critical Jeans wave number. Chandrasekhar (1961) used collision dominated magnetohydrodynamic (MHD) equations to study the effect of magnetic field and rotation on the gravitational instability and found that both magnetic field and rotation leave the Jeans wave number unaltered. The effect of rotation on the onset of gravitational collapse and the growth rate of magnetorotational 
instability of a finite electrically conducting viscoelastic medium under both strongly and weakly coupled plasma limits for transverse and longitudinal modes of wave propagation is investigated by Dhiman and Sharma (2014) and found that rotation reduces the growth rate of Jeans instability. It has been shown that the magnetorotational instability plays a crucial role in driving plasma turbulence in accretion disks and is believed to be responsible for angular momentum transport, which solves a long standing puzzle how the materials in the accretion disks fall inward to feed the stars or the black holes in the center (Velikhov, 1959; Chandrasekhar, 1960). In many space and astrophysical situations the plasma is collisionless. However, the fluid description for a rarefied plasma in a magnetic field can be used, since a strong magnetic field can replace the randomizing role of collisions and the collisional isotropic pressure becomes anisotropic pressure tensor. In such situations the Chew et al. (1956) equations are used with certain limitations when the heat flux vector is neglected and lead to different kinds of waves and instabilities like the Alfven, slow and fast MHD waves as well as the firehose and mirror instabilities. The Chew et al. (1956) equations are derived by taking velocity moments of the collisionless Boltzmann transport equation assuming that the thermal heat flow along the field lines can be neglected. Considering the importance of anisotropic pressure plasma, various investigations have been carried out by the authors listed in the references (Gliddon, 1966; Kalra et al., 1970; Kathuria and Kalra, 1973; Chhajlani and Purohit, 1985; Yajima, 1966; Summers, 1978; Ferriere, 2004; Shrauner, 1967; Gedalin, 1993; Gebretsadkan and Kalra, 2002; Ghildyal and Kalra, 1997; Chust and Belmont, 2006), all of them using double adiabatic Chew et al. (1956) equations neglecting the heat flux vector.

Gravitational instability on propagation of magnetohydrodynamic (MHD) waves in astrophysical plasma is investigated by Alemayehu and Tessema (2013a) by considering the effect of gravitational instability and viscosity with anisotropic pressure tensor and heat conducting plasma. Their results indicate that the transverse propagation is affected by the inclusion of heat conduction. For parallel propagation, they showed that the firehose mode to be unaffected whereas the mode corresponding to the gravitational instability in astrophysical plasma to be modified by anisotropic pressure tensor being stable in the presence of viscosity and strong magnetic field at considerable wavelengths. Alemayehu and Tessema (2013b) also studied the effect of viscosity on propagation of MHD waves in astrophysical plasma by considering the 
effect of viscosity with anisotropic pressure tensor. Their results show that an astrophysical plasma with anisotropic pressure tensor to be stable in the presence of viscosity and strong magnetic field at considerable wavelengths.

The Chew et al. (1956) model has been extended by Whang (1971) to include the heat flux vector which appears in the higher velocity moments of the collisionless Boltzmann equation. The hierarchy of the moment equations is truncated by writing the heat flux vector in terms of the parameters that appear in the lower moments. The modified Chew et al. (1956) equations that include the heat flux vector have been used by Kalra and Kathuria (1979); Namikawa and Hamabata (1981) to study waves and instabilities in space plasma. It has been pointed out that higher order moments contribute terms some of which do not vanish in the limit of vanishing heat flux, and the contribution from these terms is of the same order of magnitude as the contribution from the other terms in the original Chew et al. (1956) equations. Following Whang (1971), the equations for a self-gravitating, collisionless, heat conducting plasma were used by Singh and Kalra (1986) to investigate the influence of these additional terms. They found that the gravitational instability sets in at a comparatively shorter wavelength and the growth rate is enhanced owing to the inclusion of these terms in the case of parallel propagation and the condition for instability remained unaltered when the direction of propagation is transverse to the direction of magnetic field. The self-gravitational instability of rotating anisotropic heatconducting plasma with modified Chew et al. (1956) equations is investigated (Prajapati et al., 2008; Ren et al., 2011).

In all of the above studies, the plasma has been considered to be initially at rest. But, in some space and astrophysical situations (e.g., movement of the ionosphere, motion of the plasma along magnetic lines, gross motion of the surface of the sun and stars, motion of gaseous clouds in the interstellar medium, etc.), the plasma may also be initially drifting in a certain direction. The socalled "Dust Devil", which is gravitating, rotating and drifting that is observed here on earth and even on Mars, may simulate this situation. Thus, in the present work, we derive the dispersion relation of a gravitating, rotating, heat conducting and drifting anisotropic plasma.

The methodology we used here assumes a medium of an inviscid, unbounded, collisionless, gravitating and rotating heat conducting anisotropic drifting plasma which is not, to our knowledge considered by others. The medium is assumed to be embedded in a strong magnetic 
field oriented along the $\mathrm{z}$-axis. The plasma is supposed to drift with a uniform velocity $\mathrm{v}_{0}$ along the direction of the magnetic field. The strong magnetic field replaces the randomizing role of collisions so that the fluid descriptions for a rarefied plasma can be used. In such assumptions the solution of the dispersion relation is investigated using the analytical approach as the general dispersion relation is too complicated with the inclusion of drift velocity. A sample of polar plot is drawn to illustrative phase speed variations with drift velocity using FORTRAN program.

The basic equations of the MHD model for a gravitating, rotating, and heat conducting inviscid plasma which is drifting are presented below. The general dispersion relation of the problem is derived in section 3. The dispersion relation is checked with similar previous results when the drift corrections are waved and discussed in several limiting cases analytically in section 4 .

\section{BASIC EQUATIONS OF THE PROBLEM}

We consider a medium of an inviscid, unbounded, collisionless, gravitating and rotating heat conducting anisotropic plasma which is drifting. The medium is assumed to be embedded in a strong magnetic field oriented along the z-axis. The plasma is supposed to drift with a uniform velocity $\mathrm{v}_{0}$ along the direction of the magnetic field. The strong magnetic field replaces the randomizing role of collisions so that the fluid descriptions for a rarefied plasma can be used. In such assumptions the basic closed set of equations of the problem are written as follows.

The equation of motion of the system is written as

$\rho \frac{\mathrm{dv}}{\mathrm{dt}}+\nabla \cdot \mathrm{P}-\frac{1}{4 \pi}(\nabla \times \mathrm{B}) \times \mathrm{B}-\rho \nabla \phi-2 \rho(\mathrm{v} \times \Omega)=0$,

The continuity equation reads as

$$
\frac{\partial \rho}{\partial \mathrm{t}}+\nabla \cdot(\rho \mathrm{v})=0
$$

Under the assumption of perfect conductivity, Ohm's law together with Faraday's law gives the following induction equation

$$
\frac{\partial \mathrm{B}}{\partial \mathrm{t}}=\nabla \times(\mathrm{v} \times \mathrm{B})
$$


Poisson equation for gravitational potential $\phi$ is written as

$$
\nabla^{2} \phi=-4 \pi G \rho \text {. }
$$

The evolutions of the parallel and perpendicular pressures and heat flux vectors are described, respectively (Whang, 1971; Prajapati et al., 2008) as

$\frac{\mathrm{d}}{\mathrm{dt}}\left(\frac{\mathrm{B}^{2} \mathrm{P}_{\|}}{\rho^{3}}\right)=-\frac{2 \mathrm{~B}^{2}}{\rho^{3}} \hat{\mathrm{n}} \cdot \nabla\left(\frac{\mathrm{q}_{\|}}{\mathrm{B}}\right)$,

$\frac{\mathrm{d}}{\mathrm{dt}}\left(\frac{\mathrm{P}_{\perp}}{\rho \mathrm{B}}\right)=-\frac{1}{\rho} \hat{\mathrm{n}} \cdot \nabla\left(\frac{\mathrm{q}_{\perp}}{\mathrm{B}}\right)$,

$\frac{d}{d t}\left(\frac{B^{3} q_{\|}}{\rho^{4}}\right)=\frac{3 B^{2}}{2 \rho^{3}} \hat{n} \cdot\left[\frac{P_{\|} P_{\perp}}{\rho^{2}}(\nabla B)-B \frac{P_{\|}}{\rho} \nabla\left(\frac{P_{\|}}{\rho}\right)\right]$,

$\frac{\mathrm{d}}{\mathrm{dt}}\left(\frac{\mathrm{q}_{\perp}}{\rho^{2}}\right)=\frac{1}{\rho \mathrm{B}} \hat{\mathrm{n}} \cdot\left[\frac{\mathrm{P}_{\perp}^{2}}{\rho^{2}}(\nabla \mathrm{B})-\mathrm{B} \frac{\mathrm{P}_{\|}}{\rho} \nabla\left(\frac{\mathrm{P}_{\perp}}{\rho}\right)\right]$,

Where, $\hat{\mathrm{n}}=\mathbf{B} / \mathrm{B}$ is the unit vector along the magnetic field, $\rho$ the plasma density, $\frac{\mathrm{d}}{\mathrm{dt}}=\frac{\partial}{\partial \mathrm{t}}+\mathrm{v} \cdot \nabla$ the convective derivative, $\mathbf{v}$ the fluid velocity, $\mathbf{B}$ the magnetic field, $\phi$ the gravitational potential, and $\mathrm{P}$ denotes the anisotropic pressure tensor defined as $\mathrm{P}=\mathrm{P}_{\perp} \mathrm{I}+\left(\mathrm{P}_{\|}-\mathrm{P}_{\perp}\right) \hat{n} \hat{n}$,

with $\mathbf{I}$ the unit tensor. $\mathrm{P}_{\perp}, \mathrm{P}_{\|}, \mathrm{q}_{\perp}$, and $\mathrm{q}_{\|}$represent, respectively, the components of pressure and heat flux perpendicular and parallel to the magnetic field. $\mathrm{G}$ is the gravitational constant and $\Omega=$ $\Omega\left(\Omega_{\mathrm{X}}, 0, \Omega_{\mathrm{Z}}\right)$ represents the rotational frequency of the system.

\section{LINEARIZATION AND DERIVATION OF THE GENERAL DISPERSION RELATION}

In the linearization, we write the space and time dependent physical quantities $\rho, v, B, P, q$, and $\phi$, in the form of the sum of an equilibrium and perturbed part as

$\rho=\rho_{0}+\rho^{\prime}, v=v_{0}+v^{\prime}, B=B_{0}+b$, etc.

The perturbed quantities are assumed to be small compared to the equilibrium values and the equilibrium values are supposed to be uniform in space and time. Using these assumptions, the linearized form of the Equations (1)-(8) become: 


$$
\begin{aligned}
& \left(\frac{\partial}{\partial t}+\boldsymbol{v}_{\mathbf{0}} \cdot \boldsymbol{\nabla}\right) v^{\prime}+\nabla\left(P_{\perp}^{\prime}+\frac{\mathbf{B}_{\mathbf{0}} \cdot \mathbf{b}}{4 \pi}\right) \\
& -\left(\boldsymbol{B}_{\mathbf{0}} \cdot \boldsymbol{\nabla}\right)\left[\frac{P_{\perp}^{0}-P_{\|}^{0}+B_{\frac{0}{4 \pi}}^{2}}{B_{0}^{2}} \mathbf{b}-2 \mathrm{~B}_{0}\left(P_{\perp}^{0}-P_{\|}^{0}\right) \frac{\mathbf{B}_{\mathbf{0}} \cdot \mathbf{b}}{4 \pi}+B_{0} \frac{P_{\perp}^{\prime}-P_{\|}^{\prime}}{B_{0}^{2}}\right]- \\
& \rho_{-}\{0\} \nabla \phi^{\prime}-2\left[\left(v_{-} 0 \times \Omega\right) \rho^{\prime}+\rho_{-}\{0\}\left(\mathbf{v}^{\prime} \times \mathbf{\Omega}\right)\right]=0, \\
& \left(\frac{\partial}{\partial \mathrm{t}}+\mathrm{v}_{0} \cdot \nabla\right) \rho^{\prime}+\rho_{0} \nabla \cdot \mathrm{v}^{\prime}=0 \\
& \frac{\partial \mathrm{b}}{\partial \mathrm{t}}=\nabla \times\left[\mathrm{v}_{0} \times \mathrm{b}+\mathrm{v}^{\prime} \times \mathrm{B}_{0}\right] \text {, } \\
& \nabla^{2} \phi^{\prime}=-4 \pi \mathrm{G} \rho{ }^{\prime}, \\
& \left(\frac{\partial}{\partial \mathrm{t}}+\mathrm{v}_{0} \cdot \nabla\right)\left[\mathrm{P}_{\|}^{\prime}+\frac{2 \mathrm{P}_{\|}^{0}}{\mathrm{~B}_{0}} \mathrm{~b}_{\mathrm{z}}-\frac{2 \mathrm{P}_{\|}^{0}}{\rho_{0}} \rho^{\prime}\right]=-2 \hat{\mathrm{n}} \cdot \nabla \mathrm{q}_{\|}^{\prime}+\frac{2 \mathrm{q}_{\|}^{0}}{\mathrm{~B}_{0}} \hat{\mathrm{n}} \cdot \nabla \mathrm{b}_{\mathrm{z}}, \\
& \left(\frac{\partial}{\partial \mathrm{t}}+\mathrm{v}_{0} \cdot \nabla\right)\left[\mathrm{P}_{\perp}^{\prime}-\frac{\mathrm{P}_{\perp}^{0}}{\rho_{0}} \rho^{\prime}-\frac{\mathrm{P}_{\perp}^{0}}{\mathrm{~B}_{0}} \mathrm{~b}_{\mathrm{z}}\right]=-\hat{\mathrm{n}} \cdot \nabla \mathrm{q}_{\perp}^{\prime}+\frac{\mathrm{q}_{\perp}^{0}}{\mathrm{~B}_{0}} \hat{\mathrm{n}} \cdot \nabla \mathrm{b}_{\mathrm{z}} \\
& \left(\frac{\partial}{\partial \mathrm{t}}+\mathrm{v}_{0} \cdot \nabla\right)\left[\mathrm{q}_{\|}^{\prime}+\frac{3 \mathrm{q}_{\|}^{0}}{\mathrm{~B}_{0}} \mathrm{~b}_{\mathrm{z}}-\frac{4 \mathrm{q}_{\|}^{0}}{\rho_{0}} \rho^{\prime}\right]=-\frac{3}{2} \hat{\mathrm{n}} \cdot\left(\frac{\mathrm{P}_{\|}^{0} \mathrm{P}_{\perp}^{0}}{\rho_{0} \mathrm{~B}_{0}} \nabla \mathrm{b}_{\mathrm{z}}-\frac{\mathrm{P}_{\|}^{0}}{\rho_{0}} \nabla \mathrm{P}_{\|}^{\prime}+\frac{\mathrm{P}_{\| 0}^{2}}{\rho_{0}^{2}} \nabla \rho^{\prime}\right), \\
& \left(\frac{\partial}{\partial \mathrm{t}}+\mathrm{v}_{0} \cdot \nabla\right)\left[\mathrm{q}_{\perp}^{\prime}-\frac{2 \mathrm{q}_{\perp}^{0}}{\rho_{0}} \rho^{\prime}\right]=\frac{\mathrm{P}_{\perp 0}^{2}}{\mathrm{~B}_{0} \rho_{0}} \hat{\mathrm{n}} \cdot \nabla \mathrm{b}_{\mathrm{z}}-\frac{\mathrm{P}_{\|}^{0}}{\rho_{0}} \hat{\mathrm{n}} \cdot \nabla \mathrm{P}_{\perp}^{\prime}+\frac{\mathrm{P}_{\|}^{0} \mathrm{P}_{\perp}^{0}}{\rho_{0}^{2}} \hat{\mathrm{n}} \cdot \nabla \rho^{\prime} .
\end{aligned}
$$

In the absence of heat flux vector, equations (13) and (14) reduce to the usual double adiabatic Chew et al. (1956) equations. Since, the higher order moments give some terms that are of the same order of magnitude as the other terms in the Chew et al. (1956) equations (Singh and Kalra, 1986; Prajapati et al., 2008) and these terms persist in the limit of zero heat flux, we multiply equations (15) and (16) by $\alpha$ to distinguish those terms which will arise in the dispersion relation due to the presence of heat flux vector but do not contain it explicitly. The value of $\alpha$ will be taken to be unity in the presence of heat flux vector and zero otherwise.

In an unbounded homogeneous medium, any arbitrary perturbation can be Fourier analyzed and written in terms of plane waves of frequency $\omega$ and wave vector $\mathbf{k}$. Since the unperturbed magnetic field has been chosen to be directed along the z-axis, the wave vector can be taken, without loss of generality, in the xz-plane. The harmonic space and time dependence of the perturbed quantities may thus be written as

$\propto \exp \left[\mathrm{i}\left(\mathrm{k}_{\perp} \mathrm{x}+\mathrm{k}_{\|} \mathrm{z}-\omega \mathrm{t}\right)\right]$, 
Where, the wave vector $\mathrm{k}\left(\mathrm{k}_{\perp}, 0, \mathrm{k}_{\|}\right)$) is taken to be real. When the space-time dependence of the perturbations given by Equation (17) is applied to the set of linearized equations (9) to (16), three linear algebraic equations in terms of the amplitude components $\mathrm{v}_{1}, \mathrm{v}_{2}, \mathrm{v}_{3}$, (where 1,2 , 3 , represent $x, y, z$ ) are obtained. In matrix form these can be written as

$\mathrm{A}_{\mathrm{ij}} \mathrm{V}_{\mathrm{j}}=0 \quad \mathrm{i}, \mathrm{j}=1,2,3$

The components of the matrix $A_{i j}$ are written as follows:

$$
\begin{gathered}
A_{11}=\left(\omega^{2}-k_{\|}^{2} s_{\|}^{2} \alpha\right)\left(\omega^{\prime 2}+k_{\|}^{2} A^{2}-k_{\perp}^{2} v_{a}^{2}+4 \pi G \rho \frac{k_{\perp}^{2}}{k^{2}}\right)-k_{\perp}^{2}\left(2 s_{\perp}^{2} \omega^{\prime 2}+\frac{q_{\perp}}{\rho} k_{\|} \omega^{\prime}-k_{\|}^{2} s_{\perp}^{2} Q^{2} \alpha\right) \\
A_{12}=-2 i \Omega_{z} \omega^{\prime}\left(\omega^{\prime 2}-k_{\|}^{2} s_{\|}^{2} \alpha\right), \\
\mathrm{A}_{13}=\mathrm{k}_{\|} \mathrm{k}_{\perp}\left[\frac{4 \pi \mathrm{G} \rho}{\mathrm{k}^{2}}\left(\omega^{\prime 2}-\mathrm{k}_{\|}^{2} \mathrm{~s}_{\|}^{2} \alpha\right)-\mathrm{s}_{\perp}^{2} \omega^{2}-\frac{2 \mathrm{q}_{\perp}}{\rho} \mathrm{k}_{\|} \omega^{\prime}+\mathrm{k}_{\|}^{2} \mathrm{~s}_{\|}^{2} \mathrm{~s}_{\perp}^{2} \alpha\right], \\
A_{21}=-2 i\left(k_{\perp} v_{0} \Omega_{x}-\Omega_{Z} \omega^{\prime}\right), \\
\mathrm{A}_{22}=\omega^{2}+\mathrm{k}_{\|}^{2} \mathrm{~A}^{2}, \\
A_{23}=-2 i \Omega_{x}\left(k_{\|} v_{0}+\omega^{\prime}\right), \\
A_{31}=k_{\|} k_{\perp}\left[\left(\omega^{\prime 2}-3 k_{\|}^{2} s_{\|}^{2} \alpha\right)\left(\frac{4 \pi G \rho}{k^{2}}-s_{\perp}^{2}+s_{\|}^{2}\right)-s_{\|}^{2} \omega^{\prime 2}+3 k_{\|}^{2} s_{\|}^{2} Q^{2} \alpha\right], \\
A_{32}=2 i \Omega_{x} \omega^{\prime}\left(\omega^{2}-3 k_{\|}^{2} s_{\|}^{2} \alpha\right), \\
A_{33}=\left(\omega^{\prime 2}+4 \pi G \rho \frac{k_{\|}^{2}}{k^{2}}\right)\left(\omega^{\prime 2}-3 k_{\|}^{2} s_{\|}^{2} \alpha\right)-k_{\|}^{2}\left(3 s_{\|}^{2} \omega^{\prime 2}+8 k_{\|} \frac{q_{\|}}{\rho} \omega^{\prime}-3 k_{\|}^{2} s_{\|}^{4} \alpha\right) .
\end{gathered}
$$

The above expressions are written by using the following notations:

$\omega^{\prime}=\omega-\mathrm{k}_{\|} \mathrm{v}_{0} ;$ the Doppler shifted frequency,

$s_{\|, \perp}^{2}=\frac{P_{\|, \perp}}{\rho} ;$ the parallel, perpendicular sound speeds,

$v_{a}^{2}=\frac{B^{2}}{4 \pi \rho} ;$ the Alfven velocity,

$\mathrm{A}^{2}=\mathrm{s}_{\|}^{2}-\mathrm{s}_{\perp}^{2}-\mathrm{v}_{\mathrm{a}}^{2} \mathrm{a}, \quad \mathrm{Q}^{2}=\mathrm{s}_{\|}^{2}+\mathrm{s}_{\perp}^{2}, \quad \mathrm{k}^{2}=\mathrm{k}_{\|}^{2}+\mathrm{k}_{\perp}^{2}$.

As usual, the set of equation given by equation (18) has a non-trivial solution if the determinant $\left|A_{i j}\right| \mid$ vanishes producing the following general dispersion relation: 
$\left[\left(\omega^{\prime}-\mathrm{k}_{\|}^{2} \mathrm{~s}_{\|}^{2} \alpha\right)\left(\omega^{\prime 2}+\mathrm{k}_{\|}^{2} \mathrm{~A}^{2}-\mathrm{k}_{\perp}^{2} \mathrm{v}_{\mathrm{a}}^{2}+4 \pi \mathrm{G} \rho \frac{\mathrm{k}_{\perp}^{2}}{\mathrm{k}^{2}}\right)-\mathrm{k}_{\perp}^{2}\left(2 \mathrm{~s}_{\perp}^{2} \omega^{2}+\frac{\mathrm{q}_{\perp}}{\rho} \mathrm{k}_{\|} \omega^{\prime}-\mathrm{k}_{\|}^{2} \mathrm{~s}_{\perp}^{2} \mathrm{Q}^{2} \alpha\right)\right] \times$

$\left\{\left(\omega^{\prime 2}+k_{\|}^{2} A^{2}\right)\left[\left(\omega^{\prime 2}+4 \pi G \rho \frac{k_{\|}^{2}}{k^{2}}\right)\left(\omega^{2}-3 k_{\|}^{2} s_{\|}^{2} \alpha\right)-k_{\|}^{2}\left(3 s_{\|}^{2} \omega^{\prime 2}+\right.\right.\right.$

$\left.\left.\left.8 \mathrm{k}_{\|} \frac{\mathrm{q}_{\|}}{\rho} \omega^{\prime}-3 \mathrm{k}_{\|}^{2} \mathrm{~s}_{\|}^{4} \alpha\right)\right]-4 \Omega_{\mathrm{x}}^{2}\left(\mathrm{k}_{\|} \mathrm{v}_{0} \omega^{\prime}+\omega^{\prime 2}\right)\left(\omega^{\prime 2}-3 \mathrm{k}_{\|}^{2} \mathrm{~s}_{\|}^{2} \alpha\right)\right\}-4 \Omega_{\mathrm{z}}\left(\omega^{\prime 2}-\mathrm{k}_{\|}^{2} \mathrm{~s}_{\|}^{2} \alpha\right)\left\{\mathrm{k}_{\|} \mathrm{k}_{\perp} \Omega_{\mathrm{x}}\left(\mathrm{k}_{\|} \mathrm{v}_{0} \omega^{\prime}+\right.\right.$

$\left.\omega^{\prime 2}\right) \times\left[\left(\omega^{\prime 2}-3 k_{\|}^{2} s_{\|}^{2} \alpha\right)\left(\frac{4 \pi G \rho}{k^{2}}-s_{\perp}^{2}+s_{\|}^{2}\right)-s_{\|}^{2} \omega^{\prime 2}+3 k_{\|}^{2} s_{\|}^{2} Q^{2} \alpha\right]-\left(k_{\perp} v_{0} \Omega_{X} \omega^{\prime}-\Omega_{z} \omega^{\prime 2}\right) \times$

$\left.\left[\left(\omega^{\prime 2}+4 \pi G \rho \frac{\mathrm{k}_{\|}^{2}}{\mathrm{k}^{2}}\right)\left(\omega^{\prime 2}-3 \mathrm{k}_{\|}^{2} \mathrm{~s}_{\|}^{2} \alpha\right)-\mathrm{k}_{\|}^{2}\left(3 \mathrm{~s}_{\|}^{2} \omega^{2}+8 \mathrm{k}_{\|} \frac{\mathrm{q}_{\|}}{\rho} \omega^{\prime}-3 \mathrm{k}_{\|}^{2} \mathrm{~s}_{\|}^{4} \alpha\right)\right]\right\}+$

$\mathrm{k}_{\|} \mathrm{k}_{\perp}\left[\frac{4 \pi \mathrm{G} \rho}{\mathrm{k}^{2}}\left(\omega^{\prime 2}-\mathrm{k}_{\|}^{2} \mathrm{~s}_{\|}^{2} \alpha\right)-\mathrm{s}_{\perp}^{2} \omega^{\prime 2}-\frac{2 \mathrm{q}_{\perp}}{\rho} \mathrm{k}_{\|} \omega^{\prime}+\mathrm{k}_{\|}^{2} \mathrm{~s}_{\|}^{2} \mathrm{~s}_{\perp}^{2} \alpha\right] \times$

$\left\{4 \Omega_{\mathrm{x}}\left(\mathrm{k}_{\perp} \mathrm{v}_{0} \Omega_{\mathrm{x}} \omega^{\prime}-\Omega_{\mathrm{z}} \omega^{\prime 2}\right)\left(\omega^{\prime 2}-3 \mathrm{k}_{\|}^{2} \mathrm{~s}_{\|}^{2} \alpha\right)-\mathrm{k}_{\|} \mathrm{k}_{\perp}\left(\omega^{\prime 2}+\mathrm{k}_{\|}^{2} \mathrm{~A}^{2}\right)\left[\left(\omega^{\prime 2}-3 \mathrm{k}_{\|}^{2} \mathrm{~s}_{\|}^{2} \alpha\right)\left(\frac{4 \pi \mathrm{G} \rho}{\mathrm{k}^{2}}-\mathrm{s}_{\perp}^{2}+\right.\right.\right.$

$\left.\left.\left.s_{\|}^{2}\right)-s_{\|}^{2} \omega^{2}+3 k_{\|}^{2} s_{\|}^{2} Q^{2} \alpha\right]\right\}=0$

\section{DISCUSSION OF THE DISPERSION RELATION}

\subsection{Agreement with the literature}

When the drift velocity corrections are not considered (i.e., $v_{0}=0$, $\omega^{\prime}=\omega-\mathrm{k}_{\|} \mathrm{v}_{0}=\omega$ ), the general dispersion relation given by Equation(19) becomes :

$\left[\left(\omega^{2}-k_{\|}^{2} s_{\|}^{2} \alpha\right)\left(\omega^{2}+k_{\|}^{2} A^{2}-k_{\perp}^{2} v_{a}^{2}+4 \pi G \rho \frac{k_{\perp}^{2}}{k^{2}}\right)-k_{\perp}^{2}\left(2 s_{\perp}^{2} \omega^{2}+\frac{q_{\perp}}{\rho} k_{\|} \omega-k_{\|}^{2} s_{\perp}^{2} Q^{2} \alpha\right)\right] \times\left\{\left(\omega^{2}+\right.\right.$

$\left.\mathrm{k}_{\|}^{2} \mathrm{~A}^{2}\right)\left[\left(\omega^{2}+4 \pi \mathrm{G} \rho \frac{\mathrm{k}_{\|}^{2}}{\mathrm{k}^{2}}\right)\left(\omega^{2}-3 \mathrm{k}_{\|}^{2} \mathrm{~s}_{\|}^{2} \alpha\right)-\mathrm{k}_{\|}^{2}\left(3 \mathrm{~s}_{\|}^{2} \omega^{2}+\right.\right.$

$\left.\left.\left.8 \mathrm{k}_{\|} \frac{\mathrm{q}_{\|}}{\rho} \omega-3 \mathrm{k}_{\|}^{2} \mathrm{~s}_{\|}^{4} \alpha\right)\right]-4 \Omega_{\mathrm{x}}^{2} \omega^{2}\left(\omega^{2}-3 \mathrm{k}_{\|}^{2} \mathrm{~s}_{\|}^{2} \alpha\right)\right\}-4 \Omega_{\mathrm{z}}\left(\omega^{2}-\mathrm{k}_{\|}^{2} \mathrm{~s}_{\|}^{2} \alpha\right)\left\{\mathrm{k}_{\|} \mathrm{k}_{\perp} \Omega_{\mathrm{x}} \omega^{2} \times\right.$

$\left[\left(\omega^{2}-3 k_{\|}^{2} s_{\|}^{2} \alpha\right)\left(\frac{4 \pi G \rho}{k^{2}}-s_{\perp}^{2}+s_{\|}^{2}\right)-s_{\|}^{2} \omega^{2}+3 k_{\|}^{2} s_{\|}^{2} Q^{2} \alpha\right]+\Omega_{z} \omega^{2}\left[\left(\omega^{2}+\right.\right.$

$\left.\left.\left.4 \pi G \rho \frac{\mathrm{k}_{\|}^{2}}{\mathrm{k}^{2}}\right)\left(\omega^{2}-3 \mathrm{k}_{\|}^{2} \mathrm{~s}_{\|}^{2} \alpha\right)-\mathrm{k}_{\|}^{2}\left(3 \mathrm{~s}_{\|}^{2} \omega^{2}+8 \mathrm{k}_{\|} \frac{\mathrm{q}_{\|}}{\rho} \omega-3 \mathrm{k}_{\|}^{2} \mathrm{~s}_{\|}^{4} \alpha\right)\right]\right\}+$

$\mathrm{k}_{\|} \mathrm{k}_{\perp}\left[\frac{4 \pi \mathrm{G} \rho}{\mathrm{k}^{2}}\left(\omega^{2}-\mathrm{k}_{\|}^{2} \mathrm{~s}_{\|}^{2} \alpha\right)-\mathrm{s}_{\perp}^{2} \omega^{2}-\frac{2 \mathrm{q}_{\perp}}{\rho} \mathrm{k}_{\|} \omega+\mathrm{k}_{\|}^{2} \mathrm{~s}_{\|}^{2} \mathrm{~s}_{\perp}^{2} \alpha\right] \times\left\{4 \Omega_{\mathrm{x}} \Omega_{\mathrm{z}} \omega^{2}\left(\omega^{2}-3 \mathrm{k}_{\|}^{2} \mathrm{~s}_{\|}^{2} \alpha\right)-\mathrm{k}_{\|} \mathrm{k}_{\perp}\left(\omega^{2}+\right.\right.$

$\left.\left.\mathrm{k}_{\|}^{2} \mathrm{~A}^{2}\right)\left[\left(\omega^{2}-3 \mathrm{k}_{\|}^{2} \mathrm{~s}_{\|}^{2} \alpha\right)\left(\frac{4 \pi \mathrm{G} \rho}{\mathrm{k}^{2}}-\mathrm{s}_{\perp}^{2}+\mathrm{s}_{\|}^{2}\right)-\mathrm{s}_{\|}^{2} \omega^{2}+3 \mathrm{k}_{\|}^{2} \mathrm{~s}_{\|}^{2} \mathrm{Q}^{2} \alpha\right]\right\}=0$.

This dispersion relation is similar to the one derived by? We would like to point out here that it seems there is an error in their dispersion relation that they missed some terms in their dispersion (C) CNCS, Mekelle University 
relation given by their equation number 22. All their subsequent discussions are correct because they discussed the transverse and parallel propagation cases separately. Their dispersion relation becomes incorrect due to the missed terms, when one considers the case of oblique propagation where both $\mathrm{k}_{\perp}$ and $\mathrm{k}_{\|}$are different from zero.

In the absence of drift velocity and rotation the dispersion relation derived in the present work exactly agrees with the one found by? When drift, rotation and heat flux vectors are all ignored, the dispersion relation reduces to the one derived by? We note here also that in Gliddon's dispersion relation the Alfven mode is not shown in the derivation of the dispersion relation; we know in such situations that one has a 6th order in $\omega$ equation which yields three MHD waves (slow, Alfven, and fast waves).

The general dispersion relation of the present problem given by equation (19) is of tenth order polynomial (incorporating also the odd powers of $\omega$ due to the presence of drift) indicating the existence of ten wave modes. It is very difficult to separate these modes in the general case for analytical discussions in an arbitrary direction of propagation. So, we try to discuss them in various limiting cases.

\subsection{Longitudinal mode of Propagation $\left(\mathrm{k}_{\|}=\mathrm{k}, \mathrm{k}_{\perp}=0\right)$}

In the case of parallel propagation the general dispersion relation equation (19) reduces to the following form: The entropy wave mode (modified by the drift velocity) which is factored out is given by

$$
\omega^{2}-k^{2} s_{\|}^{2} \alpha=0,
$$

and the other (of eight order in $\omega^{\prime}$ ) written as

$$
\begin{aligned}
& \left(\omega^{2}+\mathrm{k}^{2} \mathrm{~A}^{2}\right)\left\{( \omega ^ { 2 } + \mathrm { k } ^ { 2 } \mathrm { A } ^ { 2 } ) \left[\left(\omega^{\prime 2}+4 \pi \mathrm{G} \rho\right)\left(\omega^{2}-3 \mathrm{k}^{2} \mathrm{~s}_{\|}^{2} \alpha\right)-\mathrm{k}^{2}\left(3 \mathrm{~s}_{\|}^{2} \omega^{2}+\right.\right.\right. \\
& \left.\left.\left.8 \mathrm{k} \frac{\mathrm{q} \|}{\rho} \omega^{\prime}-3 \mathrm{k}^{2} \mathrm{~s}_{\|}^{4} \alpha\right)\right]-4 \Omega_{\mathrm{x}}^{2}\left(\mathrm{k} \mathrm{v}_{0} \omega^{\prime}+\omega^{\prime 2}\right)\left(\omega^{\prime 2}-3 \mathrm{k}^{2} \mathrm{~s}_{\|}^{2} \alpha\right)\right\}-4 \Omega_{\mathrm{z}}^{2} \omega^{\prime 2}\left[\left(\omega^{2}+\right.\right. \\
& \left.4 \pi \mathrm{G} \rho)\left(\omega^{\prime 2}-3 \mathrm{k}^{2} \mathrm{~s}_{\|}^{2} \alpha\right)-\mathrm{k}^{2}\left(3 \mathrm{~s}_{\|}^{2} \omega^{2}+8 \mathrm{k} \frac{\mathrm{q}_{\|}}{\rho} \omega^{\prime}-3 \mathrm{k}^{2} \mathrm{~s}_{\|}^{4} \alpha\right)\right]=0 .
\end{aligned}
$$

The above two dispersion relations indicate that in the case of parallel propagation direction all the ten wave modes propagate being affected by the drift velocity. The wave mode given by (c) CNCS, Mekelle University 
equation (21) (which vanishes in the absence of heat flux vector) may be expanded and written in terms of $\omega$ as

$$
\omega^{2}-2 \mathrm{kv}_{0} \omega+\mathrm{k}^{2}\left(\mathrm{v}_{0}^{2}-\mathrm{s}_{\|}^{2}\right)=0
$$

The solution of this mode is given by

$$
\omega_{ \pm}=\mathrm{kv}_{0} \pm \mathrm{s}_{\|} .
$$

This wave mode represents sound speed produced by the parallel pressure modified by the drift velocity. This is a stable mode independent of rotation, gravitation and heat flux vector. The drift velocity has no effect on the stability of the wave but the phase speed of this wave mode is increased by $\mathrm{v}_{0}$ for the outgoing mode $\left(\mathrm{v}_{\mathrm{ph}}^{+}=\mathrm{v}_{0}+\mathrm{s}_{\|}\right)$and decreased for the incoming mode $\left(\mathrm{v}_{\mathrm{ph}}^{-}=\mathrm{v}_{0}-\mathrm{s}_{\|}\right)$. Equation (22) is still of eight order in $\omega^{\prime}$ which is again difficult to separate the modes for analytical discussion. So again we discuss them in a particular rotation axis.

\subsubsection{Axis of rotation transverse to the magnetic field $\left(\Omega_{\mathrm{x}}=\Omega, \Omega_{\mathrm{z}}=0\right)$}

The propagating waves along the direction of the magnetic field when the axis of rotation is chosen to be across the magnetic field may be separated in to three:

One is the wave mode given by equation (21) which we have already discussed.

The second is the anisotropic Alfven wave mode modified by the drift velocity given by

$\omega^{2}-2 \mathrm{kv}_{0} \omega+\mathrm{k}^{2}\left(\mathrm{v}_{0}^{2}+\mathrm{s}_{\|}^{2}-\mathrm{s}_{\perp}^{2}-\mathrm{v}_{\mathrm{a}}^{2}\right)=0$.

The solution of this wave mode is given by

$\mathrm{v}_{\mathrm{ph}}^{ \pm}=\mathrm{v}_{0} \pm\left(\mathrm{s}_{\perp}^{2}+\mathrm{v}_{\mathrm{a}}^{2}-\mathrm{s}_{\|}^{2}\right)^{1 / 2}$. 


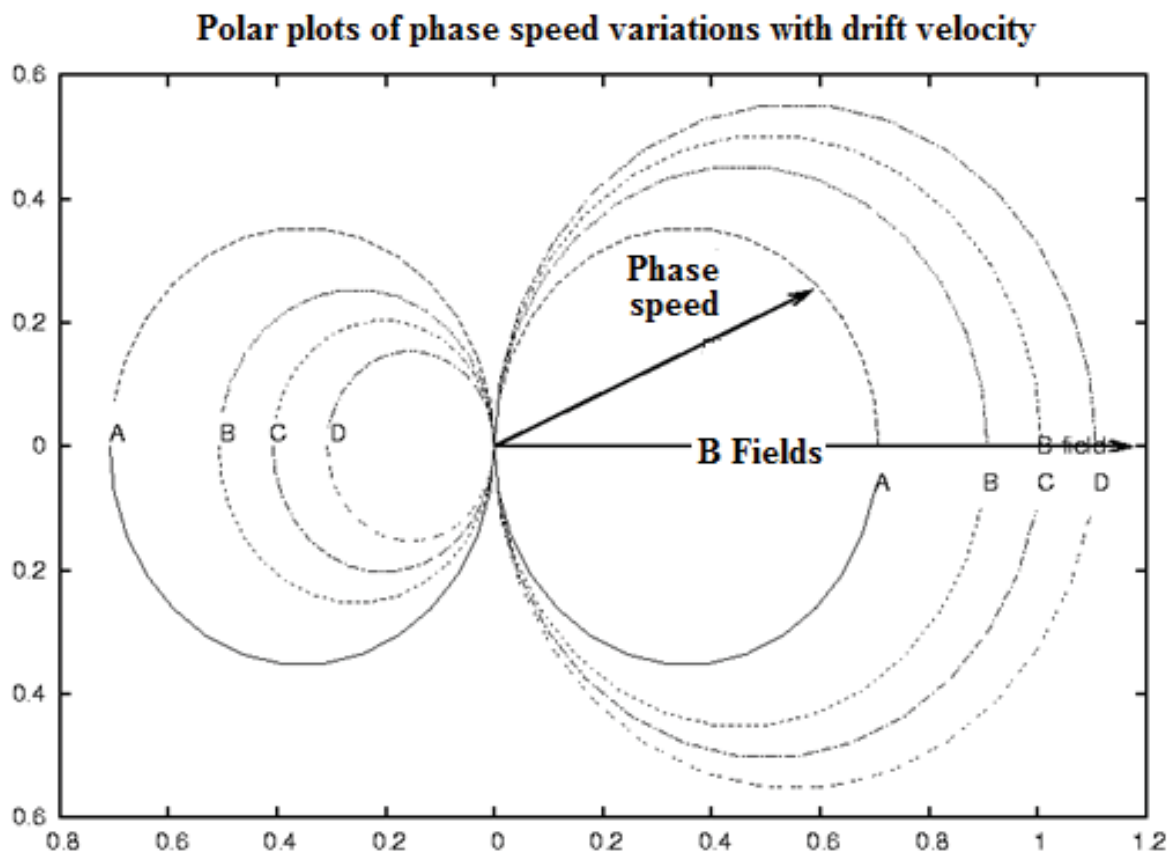

Figure 1. The polar plots of phase speed variations with drift velocity are shown for the wave mode given by equation 25. The two left and right modes label by "A" are the wave modes for $\mathrm{vo}=0$; the non-drifting case. The two left and right modes label by "B", " $\mathrm{C}$ " and "D" are the wave modes for $\mathrm{vo}^{2}=0.2,0.3$, and 0.4 , respectively. The non drifting case is shown to be symmetric, but when the drift velocity is non zero, the left and rightward propagations become anti-symmetric. It is illustrated that the phase speed of the forward propagating mode increases with increasing drift and the backward propagating mode decreases with increasing drift.

In this particular direction of propagation and axis of rotation, this wave mode is also independent of rotation, gravitation and heat flux. The firehose instability persists unaltered due to drift, but the phase speed is shifted by the drift velocity; forward propagation being increased and the backward propagation decreased by the drift velocity.

The third is still of six order in $\omega^{\prime}$ expressed as

$\left(\omega^{\prime 2}+k^{2} A^{2}\right)\left[\left(\omega^{\prime 2}+4 \pi G \rho\right)\left(\omega^{\prime 2}-3 k^{2} s_{\|}^{2} \alpha\right)-k^{2}\left(3 s_{\|}^{2} \omega^{\prime 2}+8 k \frac{q_{\|}}{\rho} \omega^{\prime}-3 k^{2} s_{\|}^{4} \alpha\right)\right]-4 \Omega_{x}^{2}\left(k v_{0} \omega^{\prime}+\right.$

$\left.\omega^{\prime 2}\right)\left(\omega^{\prime 2}-3 \mathrm{k}^{2} \mathrm{~s}_{\|}^{2} \alpha\right)=0$

This equation incorporates wave modes affected by gravitation, rotation, drift and parallel component of the heat flux. The perpendicular component of the heat flux has no effect in this particular direction of propagation and axis of rotation. 


\subsubsection{Axis of rotation parallel to the magnetic field $\left(\Omega_{\mathrm{z}}=\Omega, \Omega_{\mathrm{x}}=0\right)$}

In this case the dispersion relation expressed by Equation (22) becomes (excluding Equation (21) which is also present here) becomes

$\omega^{4}-4 \mathrm{kv}_{0} \omega^{3}+2 \mathrm{k}^{2}\left(3 \mathrm{v}_{0}^{2}+\mathrm{A}^{2}-2 \frac{\Omega^{2}}{\mathrm{k}^{2}}\right) \omega^{2}-4 \mathrm{k}^{3} \mathrm{v}_{0}\left(\mathrm{v}_{0}^{2}+\mathrm{A}^{2}-2 \frac{\Omega^{2}}{\mathrm{k}^{2}}\right) \omega+\mathrm{k}^{4}\left(\mathrm{v}_{0}^{4}+\mathrm{A}^{4}+\right.$

$\left.2 v_{0}^{2} A^{2}-4 v_{0}^{2} \frac{\Omega^{2}}{k^{2}}\right)=0$,

and

$$
\begin{aligned}
& \omega^{4}-4 \mathrm{kv}_{0} \omega^{3}+\mathrm{k}^{2}\left(6 \mathrm{v}_{0}^{2}-6 \mathrm{~s}_{\|}^{2} \alpha+4 \frac{\pi \mathrm{G} \rho}{\mathrm{k}^{2}}\right) \omega^{2}-\mathrm{k}^{3}\left(4 \mathrm{v}_{0}^{2}-2 \mathrm{v}_{0}\left(6 \mathrm{~s}_{\|}^{2} \alpha-4 \frac{\pi \mathrm{G} \rho}{\mathrm{k}^{2}}\right)-8 \frac{\mathrm{q}_{\|}}{\rho}\right) \omega+ \\
& \mathrm{k}^{4}\left(\mathrm{v}_{0}^{4}-3 \mathrm{~s}_{\|}^{2} \alpha\left(\mathrm{v}_{0}^{2}-\mathrm{s}_{\|}^{2}\right)+4 \frac{\pi \mathrm{G} \rho}{\mathrm{k}^{2}}\left(\mathrm{v}_{0}^{2}-3 \mathrm{~s}_{\|}^{2} \alpha\right)-8 \mathrm{v}_{0} \frac{\mathrm{q}_{\|}}{\rho}\right)=0 .
\end{aligned}
$$

The above two equations contain odd powers of $\omega$ due to drift velocity. Equation (28) is affected by drift velocity and rotation, but gravitation and heat flux have no effect on the wave modes. On the other hand, equation (29) is affected by drift velocity, gravitation and heat flux, but rotation has no role on these modes.

\subsection{Propagation vector transverse to the magnetic $\left(\mathrm{k}_{\|}=0, \mathrm{k}_{\perp}=\mathbf{k}\right)$}

In this case, the general dispersion relation reduces to $\left(\omega^{\prime}=\omega-\mathrm{k}_{\|} \mathrm{v}_{0}=\omega\right.$ since, $\mathrm{k}_{\|}=0$,

$\left[\omega^{2}-\mathrm{k}^{2}\left(2 \mathrm{~s}_{\perp}^{2}+\mathrm{v}_{\mathrm{a}}^{2}\right)+4 \pi \mathrm{G} \rho\right]\left(\omega^{2}-4 \Omega_{\mathrm{x}}^{2}\right)-4 \Omega_{\mathrm{z}}^{2} \omega^{2}=0$

In this particular direction of propagation, the drift velocity has no effect on the wave modes and six of the ten wave modes become degenerate. The propagating modes given by equation (30) are not affected by the drift velocity since wave normal is transverse to the direction of flow. These are identical to the wave modes discussed by? To investigate the stability of the propagating modes give by equation (30), we may again consider two cases of rotation:

\subsubsection{Axis of rotation transverse to the magnetic field $\left(\Omega_{\mathrm{x}}=\Omega, \Omega_{\mathrm{z}}=0\right)$}

When the axis of rotation is chosen to be in the perpendicular direction to the magnetic field, we obtain two propagating modes with phase speeds $\left(\mathrm{v}_{\mathrm{ph}}=\omega / \mathrm{k}\right)$ given by

$$
\begin{gathered}
\mathrm{v}_{\mathrm{ph}}^{2}=4 \frac{\Omega^{2}}{\mathrm{k}^{2}}, \\
\mathrm{v}_{\mathrm{ph}}^{2}=2 \mathrm{~s}_{\perp}^{2}+\mathrm{v}_{\mathrm{a}}^{2}-\frac{4 \pi \mathrm{G} \rho}{\mathrm{k}^{2}} .
\end{gathered}
$$


The wave modes given by equation (31) are the rotational modes with solutions

$$
\mathrm{v}_{\mathrm{ph}}= \pm 2 \Omega \text {. }
$$

These rotational modes are independent of the drift velocity, gravitation and heat flux vector and are stable. The wave modes given by Equation (32) are the gravitational modes also independent of drift velocity, rotation and heat flux vectors. The solution of these waves is given as

$$
\mathrm{v}_{\mathrm{ph}}= \pm\left(2 \mathrm{~s}_{\perp}^{2}+\mathrm{v}_{\mathrm{a}}^{2}-\frac{4 \pi \mathrm{G} \rho}{\mathrm{k}^{2}}\right)^{1 / 2}
$$

These wave modes become unstable when

$$
\frac{4 \pi G \rho}{\mathrm{k}^{2}}>2 \mathrm{~s}_{\perp}^{2}+\mathrm{v}_{\mathrm{a}}^{2}
$$

\subsubsection{Axis of rotation parallel to the magnetic field $\left(\Omega_{\mathrm{z}}=\Omega, \Omega_{\mathrm{x}}=0\right)$}

The solution of the propagating wave mode in this axis of rotation is given by

$$
\mathrm{v}_{\mathrm{ph}}= \pm\left(2 \mathrm{~s}_{\perp}^{2}+\mathrm{v}_{\mathrm{a}}^{2}+\frac{4 \Omega^{2}}{\mathrm{k}^{2}}-\frac{4 \pi \mathrm{G} \rho}{\mathrm{k}^{2}}\right)^{1 / 2}
$$

The condition for instability is given by

$$
2 \mathrm{~s}_{\perp}^{2}+\mathrm{v}_{\mathrm{a}}^{2}+\frac{4 \Omega^{2}}{\mathrm{k}^{2}}<\frac{4 \pi \mathrm{G} \rho}{\mathrm{k}^{2}}
$$

Here rotation has the stabilizing influence.

\section{CONCLUSION}

A medium of inviscid, unbounded, collisionless, gravitating, rotating and heat conducting anisotropic plasma which is drifting is considered. The medium is assumed to be embedded in a strong magnetic field oriented along the z-axis. The plasma is supposed to drift with a uniform velocity $\mathrm{v}_{0}$ along the direction of the magnetic field. The strong magnetic field replaces the randomizing role of collisions so that the fluid descriptions for a rarefied plasma is used. The basic fluid equations of the problem are linearized and the dispersion relation derived. The derived dispersion relation is compared with the dispersion relations of similar situations in the literature and some disagreements pointed out.

The dispersion relation reveals the existence of five waves modified by the drift velocity. These different wave modes are discussed in some particular cases analytically where ever possible. It is found that in the case of longitudinal propagation and rotation axis, the dispersion relation is 
factored out in to two : one affected by drift velocity and rotation while gravitation and heat flux have no effect on the wave modes and the other affected by drift velocity, gravitation and heat flux, but rotation has no role on these modes. It is also found that, in the longitudinal mode of propagation, all the five waves (modified by the drift velocity) propagate. These waves, when the axis of rotation is chosen to be across the magnetic field, are separated in to three parts: One is the modified entropy wave. The second is the anisotropic Alfven wave mode also modified by the drift velocity. It is shown that these two waves are independent of rotation, gravitation and heat flux. The drift velocity has no effect on the stability of these waves but their phase velocities are shifted; the forward propagating modes being increased and the backward propagating modes decreased. The third part incorporates three waves affected by gravitation, rotation, drift and parallel component of the heat flux. The perpendicular component of the heat flux has no effect in this particular direction of propagation and axis of rotation. When the axis of rotation is parallel to the magnetic field, besides the entropy wave, two of the waves are affected by drift velocity and rotation, but gravitation and heat flux have no effect on the waves. On the other hand, the other two waves are affected by drift velocity, gravitation and heat flux, but rotation has no role on these waves.

It is further shown that only two waves propagate in the perpendicular direction to the direction of the magnetic field. The propagating wave modes in this particular direction are not affected by the drift velocity since wave normal is transverse to the direction of flow. Such analytical discussion is limited here because of the unwieldy nature of the dispersion relation. Numerical solution of the tenth order in $\omega$ polynomial is under progress.

\section{ACKNOWLEDGEMENTS}

I would like to thank the reviewers for their constructive suggestions and the editors for their editing at the reference section.

\section{REFERENCE}

Alemayehu, M \& Tessema, S.B. 2013a. Gravitational instability on propagation of MHD waves in astrophysical plasma. J. Plasma Physics, 79: 805. 
Alemayehu, M \& Tessema, S.B. 2013b. Effect of viscosity on propagation of MHD waves in astrophysical plasma. J. Plasma Physics, 79:535.

Chandrasekhar, S. 1960. The stability of non-dissipative couette flow in hydromagnetics Proceedings, National Academy of Sciences, U.S.A, 46:253.

Chandrasekhar, S. 1961. Hydrodynamic and Hydromagnetic Stability. Oxford, Clarendon Press.

Chhajlani, R.K \& Purohit, P. 1985. Effect of hall current on the magnetogravitational instability of anisotropic plasma with generalized polytropic law. Contribution Plasma Physics, 25:615.

Chew, G.F., Goldberger, M.L \& Low, F.E. 1956. The Boltzmann Equation and the One-Fluid Hydromagnetic Equations in the Absence of Particle Collisions. Proceedings Royal Society of London, A 236:112.

Chust, T. \& Belmont, G. 2006. Closure of fluid equations in collisionless magnetoplasmas. Physics Plasmas, 13:012506.

Dhiman, J. S \& Sharma, R., 2014. Effect of rotation on the growth rate of magneto gravitational instability of a viscoelastic medium. Physics Scr., 89 :125001.

Ferriere, K.M. 2004. Low frequency linear waves and instabilities in uniform and stratified plasmas: the role of kinetic effects. Nonlinear Processes Geophysics, 11 :731.

Gebretsadkan, W.B \& Kalra, G.L. 2002. Propagation of linear waves in relativistic anisotropic magnetohydrodynamics. Physics Reviews, E 66: 057401.

Gedalin, M. 1993. Linear waves in relativistic anisotropic magneto hydrodynamics. Physics Reviews, E 47 :4354.

Ghildyal, V \& Kalra, G.L. 1997. Linear waves and instabilities in thermally anisotropic two component magneto hydrodynamics. Physics Plasmas, 4: 2044.

Gliddon, J.E.C. 1966. Gravitational instability of anisotropic plasma. Astrophysics Journal, 145: 583.

Jeans, J.H. 1902. The stability of spherical Nebula. Phil. trans. R. Soc. London, 199:1.

Kalra, G.L. Hosking, R.J \& Talwar, S.P. 1970. Effect of self-gravitation on finite ion mass on the stability of anisotropic plasma Astrophys. Space. Sci., 9: 34.

Kalra, G.L \& Kathuria, S.N. 1979. Gravitational instability of thermaly anisotropic plasma. In: IAGA Bull. No. 43. Ed: N Fukushima (Canberr: IUGG publications), 329. 
Kathuria, S.N \& Kalra, G.L. 1973. Two-stream instability in a collisionless plasma. Astrophys. Space. Sci., 24:133.

Namikawa, T \& Hamabata, H. 1981. Propagation of hydromagnetic waves through a collisionless, heat conducting plasma. J. Plasma Physics, 26: 95.

Prajapati, R.P., Parihar, A.K \& Chhajlani, R. K. 2008. Self-gravitational instability of rotating anisotropic heat-conducting plasma. Physics Plasmas, 15: 012107.

Ren, H., Cao, J., Wu, Z \& Chu, P.K. 2011. Magnetototationalinstability in a collisionless plasma with heat flux vector and anisotropic plasma with self-gravitational effect. Physics Plasmas, 18 :092117.

Summers, D. 1978. On the collisional theory of the anisotropic solar wind plasma. Solid Physics, 56 :429.

Shrauner, B.A. 1967. Propagation of hydro-magnetic waves through an anisotropic plasma. $J$. Plasma Physics, 1: 361.

Singh, B \& Kalra, G.L. 1986. Gravitational instability of thermally anisotropic plasma. Astrophysics J., 304: 6.

Velikhov, E.P. 1959. Stability of an ideally conducting liquid flowing between cylinders rotating in a magnetic field. Soviet physics, JETP, 9: 995.

Whang,Y.C. 1971. Higher moment equations and the distribution function of the solar wind plasma. Geophysics Research, 76: 07505.

Yajima, N. 1966. The effect of ion larmor radius on the propagation of magneto-acoustic waves Prog. Theor. Phys., 36:1. 